\title{
AN ESTIMATE OF THE METROLOGICAL \\ CHARACTERISTICS OF A STANDARD SAMPLE \\ OF THE COMPOSITION OF DRIED WHOLE \\ MILK USING PRIMARY AND SECONDARY \\ STATE STANDARDS
}

\author{
M. P. Krasheninina, ${ }^{1}$ M. Yu. Medvedevskikh, ${ }^{1}$ \\ S. V. Medvedevskikh, ${ }^{1}$ L. K. Neudachina, ${ }^{2}$ \\ and E. P. Sobina 1
}

UDC 543.24

A standard sample of dried whole milk GSO 9563-2010 with certified values of the mass fractions of moisture and nitrogen (protein) is developed. Its metrological characteristics, corresponding to similar world levels, are presented.

Keywords: standard sample, traceability, primary and secondary standards, mass fractions of moisture and nitrogen.

The mass fraction of protein is the most important indicator of the quality of the overwhelming majority of food products and, in particular, dried unskimmed milk. Protein enzymes catalyze a number of chemical reactions, and also albumen, which together with fats and carbohydrates, is one of the indicators, which characterize the energy value of food products, and hence its determination is essential. The requirements imposed on the mass fraction of protein are regulated, for example, in $[1,2]$.

In practice, to estimate the quality of milk and milk products, an increasing number of express analyzers, based on refractometric and ultrasonic methods as well as infrared spectroscopy, are being employed. In this situation, the need arises to increase the volume and improve the metrological facilities.

The purpose of the present paper is to set up a standard sample of the composition of dried milk with certified values of the mass fractions of nitrogen (protein) and moisture, suitable for testing, checking and calibrating different types of express analyzers, and also for monitoring the accuracy of the measurement procedure.

The Choice and Estimation of the Optimum Parameters of the Method of Determining the Mass Fraction of Nitrogen. This procedure is based on Kjeldahl's method [3], the principle of which is the quantitative conversion of organic nitrogen (the polypentide bonds of albumen) into ammonium form when heated in concentrated sulfuric acid in the presence of catalysts. Ammonia is expelled from the ammonium sulfate by concentrated alkali, which, with water vapor, is driven off into a receiver with a certain volume of boric acid. At the concluding stage, an acid-base titration with a solution of sulfuric acid is carried out to measure the nitrogen content. After the distillation is completed, the quantity of ammonia in the collect-

\footnotetext{
${ }^{1}$ Ural Research Institute of Metrology (UNIIM), Ekaterinburg, Russia; e-mail: krasheninina_m@unim.ru.

${ }^{2}$ Yeltsin Ural Federal University (UrFU), Ekaterinburg, Russia.
}

Translated from Izmeritel'naya Tekhnika, No. 9, pp. 67-71, September, 2013. Original article submitted April 19, 2013. 


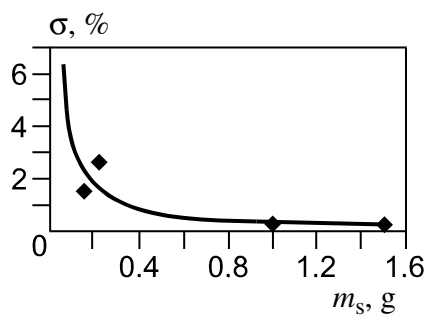

Fig. 1. Graph of the relative root mean square error $\sigma$ of the results of measurements of the mass fraction of nitrogen against the mass of the sample $m_{\mathrm{s}}$ of dried milk.

ed distillate is measured and the mass fraction of nitrogen in the material being analyzed is calculated. The mass fraction of nitrogen $W$ (as a percentage) in the sample when making measurements by Kjeldahl's method is calculated from the formula

$$
W=C M V /\left(10 m_{\mathrm{s}}\right),
$$

where $V=V_{1}-V_{0}, V_{1}$, and $V_{0}$ are the volumes of the solution of sulfuric acid, expended on the titration when analyzing the sample and in the blank test, respectively; $C$ is the molar concentration of sulfuric acid, used for the titration; $m_{\mathrm{S}}=m_{2}-m_{1}$, $m_{1}$, and $m_{2}$ are the masses of the weighing bottle and the sample and the weighing bottle, respectively; and $M$ is the atomic mass of nitrogen, $M=14.00674 \mathrm{~g} / \mathrm{mole}$.

To determine the metrological characteristics of the standard sample of the composition of dried milk, we used the State Secondary Standard of the units of mass fraction and mass (molar) concentration of the components in solid and liquid substances and materials, based on the volume titration method of analysis (henceforth, the GVET 176-1-2010) [4], having different methods of recording the final point of the titration: potentiometric, photometric and biamperometric.

An increase in the accuracy in determining the mass fraction of nitrogen by Kjeldahl's method in GVET 176-1-2010 can be achieved by optimizing the following factors: the determination of the molar concentration of the titrate, the choice of the mass of the sample and the mode of distillation, the method of recording the final titration point, and also when using methods of reproducing the mass fraction of moisture on the State Primary Standard of the unit of mass fraction and mass concentration of moisture in solid substances and materials (previously GET 173-2008) when carrying out calculations on an absolutely dry material $[5,6,7]$.

Determination of the molar concentration of the titrant - sulfuric acid. To prepare the solution of titrant, we used a titration standard of sulfuric acid of $0.05 \mathrm{M}$ with an expanded uncertainty of the certified value of $1 \%$. We determined the molar concentration of the titrant using a standard sample of sodium carbonate of the 1st category (GSO 4086-87) [8], the mass fraction of the main material in which was certified on the State Primary Standard of the units of mass (molar) fraction and mass (molar) concentration of the component in liquid and solid substances and materials based on a coulometric titration (henceforth GET 176-2010).

In order to obtain the results of a determination of the molar concentration of sulfuric acid with the least uncertainty for processing the titration curve of sodium carbonate, we used its second step and we described it by a cubic spline, which, as shown in [4], gives the most accurate results. By processing the data taking [4] into account, and also using GSO 4086-87 [8] with standardization, we were able not only to increase the accuracy with which the molar concentration of the titrant was determined by a factor of more than three, but it also enabled us to ensure the traceability of the certified value of the standard sample to GET 176-2010, in accordance with the requirements of the standard sample [9].

The choice of the optimum mass of the sample. We determined the optimum mass of the sample during the course of the experiment, during which the mass was varied from 0.1 to $1.5 \mathrm{~g}$ (Fig. 1). We chose a sample mass of $1 \mathrm{~g}$ from the best combination of the relative root mean square deviation and the decomposition time of the sample. 


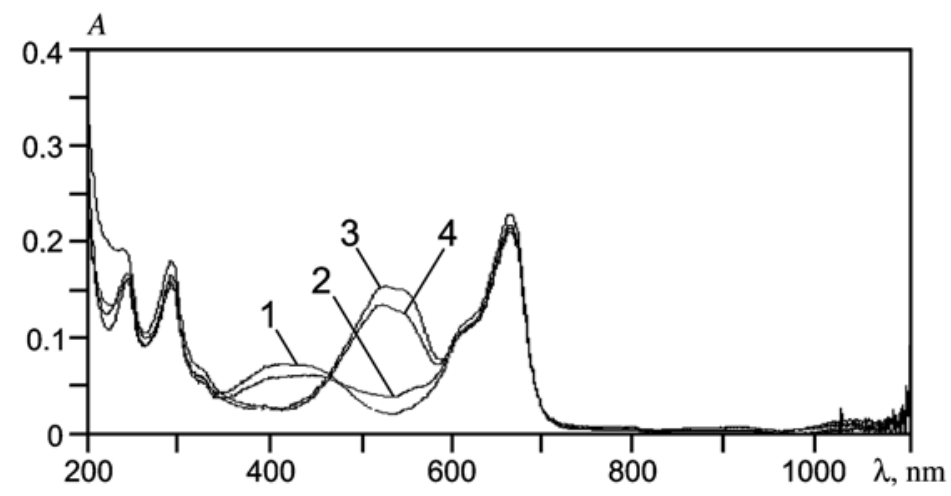

Fig. 2. Graph of the optical density $A$ of the indicator against the wavelength $\lambda$ : $1,2,3,4) p H=8.18,5.56,3.33$, and 2.50 , respectively.

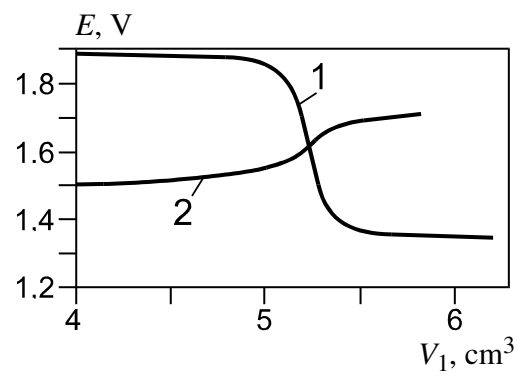

Fig. 3. Titration curves obtained using the phototrode (curve $1, \lambda=520 \mathrm{~nm}$ ) and the glass electrode (curve 2).

Choice of the distillation mode. When distilling ammonia with water vapor, it is necessary to ensure $100 \%$ extraction of the nitrogen. This procedure was carried out in an automatic S1 vapor distiller made by the Behr Labor Technick Company, forming part of the GVET 176-1-2010 sample-preparation unit, which enables the operator to specify the values of the distillation time and power. To produce the measurement conditions that ensure these requirements, we carried out an experiment to distill off ammonium sulfate (chemically pure) with a constant distillation capacity of $80 \%$ and the ability to vary the distillation time from several seconds to $10 \mathrm{~min}$, with a further determination of the volume of the distilled distillate and a degree of extraction of the nitrogen $N$, according to the formula

$$
V_{\mathrm{d}}=\left(W_{\mathrm{m}} / W_{\mathrm{c}}\right) \cdot 100
$$

where $W_{\mathrm{m}}$ is the measured mass fraction of nitrogen in the sample of ammonium sulfate; and $W_{\mathrm{c}}$ is the mass fraction of nitrogen in the sample of ammonium sulfate obtained by calculation, taking the mass fraction of the basic material in the reagent into account.

It was found that the volume of distillate distilled off can also be used as a criterion of the completeness of the extraction of the nitrogen. As a result of the experiments, we established the optimum distillation time to be 10 min, while the volume of distillate obtained is $(250 \pm 5) \mathrm{cm}^{3}$.

The choice of the optimum method of recording the final titration point. The GVET 176-1-2010 standard enables one to carry out an acid-based titration only using glass electrodes, or with a phototrode at wavelengths of 520, 555, 590, 620, and $660 \mathrm{~nm}$. The optimum wavelength, established in the phototrode, was chosen from the absorption spectra obtained in the visible region with a mixed indicator (methylene red and bromcresol green) for different value of the $\mathrm{pH}$ on a Helios spec- 


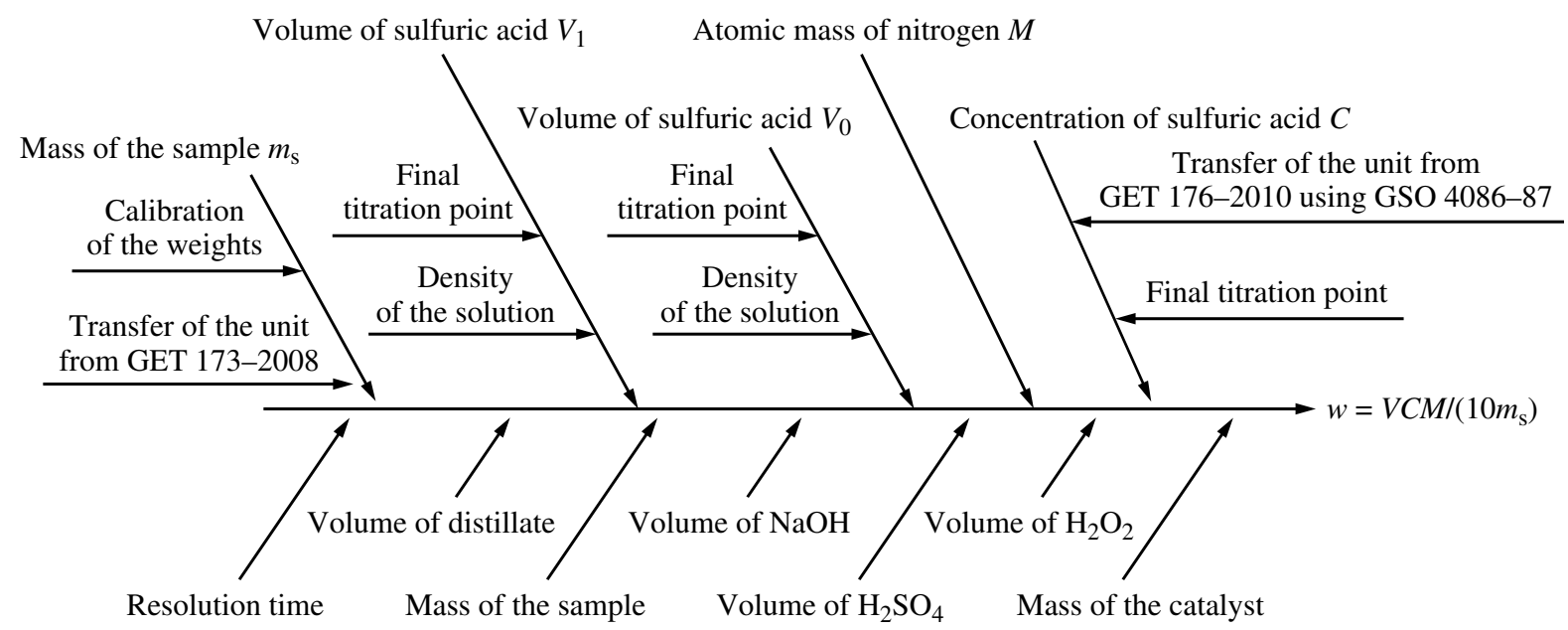

Fig. 4. Cause-effect diagram of the connections of the measured quantity and the influencing factors.

trophotometer made by Spectronic Unicam (UK) (Fig. 2). We show in Fig. 2 that the maximum difference between the optical density of the acidic and basic forms of the indicator is observed at a wavelength of $530 \mathrm{~nm}$. We chose as the optimum wavelength the one most easily acceptable for use, namely, $520 \mathrm{~nm}$.

To choose the optimum method of recording the final titration point, we analyzed the titration curves obtained using a glass electrode and a phototrode at a wavelength of $520 \mathrm{~nm}$ (Fig. 3). The jump on the titration curve with the phototrode is about $500 \mathrm{mV}$, whereas with the glass electrode it is less than $300 \mathrm{mV}$. Hence, using the phototrode we were able to increase the sensitivity of the indicator of the final titration point considerably, since the jump on the titration curve with the phototrode is more than 1.5 times greater than the jump obtained with the glass electrode.

Determination of the Metrological Characteristics of the Standard Sample. In general, according to [10, 11], the total standard uncertainty of the certified value of the standard sample is defined by the equation

$$
u_{c}(A)=\sqrt{u_{\mathrm{char}}^{2}+u_{\mathrm{h}}^{2}+u_{\mathrm{lts}}^{2}+u_{\mathrm{sts}}^{2}}
$$

where $u_{\text {char }}$ is the uncertainty of the method of characterizing the standard sample, $u_{\mathrm{h}}$ is the uncertainty arising from the nonuniformity of the material of the standard sample, and $u_{\mathrm{lts}}$ and $u_{\mathrm{sts}}$ are the uncertainties of the long-term and short-term instability of the material of the standard sample, respectively.

The expanded uncertainty of the certified value is calculated with a coverage coefficient $k=2$.

An estimate of the uncertainty of the characterization method. For completeness in taking into account the components of the uncertainty, we show in Fig. 4 a diagram of the cause-effect relations. The causes of the uncertainty of a measurement of the mass fraction of nitrogen (protein) type B, starting from (1), are measurements of the atomic mass of nitrogen, the mass of the sample, the volume of the solution and the concentration of the titrant $\left(\mathrm{H}_{2} \mathrm{SO}_{4}\right)$, and also the uncertainty in determining the final equivalence point. The contributions to the uncertainty of the measurements (see (1)), due to the equipment of the GVET 176-1-2010, are taken into account in the documentation on the standard.

Sources of uncertainty, which do not occur directly in the measurement equation, but affect the result, and are due to procedures of decomposition and distillation of the sample, were estimated by carrying out two multifactor experiments. The factors of the first experiment were the disintegration time, the volume of distilled distillate and the mass of the sample, while the factors of the second experiment are the mass of the catalyst, the volume of sulfuric acid, hydrogen peroxide and sodium hydroxide. Plans of the multifactor experiment were compiled taking $[12,13]$ into account. The adequacy of the models used is shown by the Fisher F-criterion, and a check of the significance of the regression coefficients obtained was carried out using the Student $t$-criterion. When constructing the list of uncertainties all the contributions were taken into account. 
TABLE 1. List of Uncertainties of the Results of Measurements of the Mass Fraction of Nitrogen

\begin{tabular}{|c|c|c|c|c|c|c|}
\hline Source & Estimate & $\begin{array}{c}\text { Standard } \\
\text { uncertainty } u_{i}\end{array}$ & $\begin{array}{l}\text { Sensitivity } \\
\text { coefficient } c_{i}\end{array}$ & $\begin{array}{l}\text { Distribution } \\
\text { law }\end{array}$ & $\begin{array}{c}\text { Number of degrees } \\
\text { of freedom } v_{i}\end{array}$ & $c_{i} u_{i}$ \\
\hline Mass fraction of nitrogen in the sample $W, \%$ & 4.4900 & $1.12 \cdot 10^{-2}$ & 1 & Normal & 9 & $1.12 \cdot 10^{-2}$ \\
\hline Mass of the sample and weighing bottle $m_{2}, \mathrm{~g}$ & 0.8900 & $2.89 \cdot 10^{-5}$ & -0.3637 & Uniform & $\propto$ & $-1.05 \cdot 10^{-5}$ \\
\hline Mass of the weighing bottle $m_{1}, \mathrm{~g}$ & 0.0086 & $2.89 \cdot 10^{-5}$ & 0.3637 & Ditto & $\propto$ & $1.05 \cdot 10^{-5}$ \\
\hline \multicolumn{7}{|l|}{$\begin{array}{l}\text { Volume of the solution of } \mathrm{H}_{2} \mathrm{SO}_{4} \text {, consumed in } \\
\text { the titration, } \mathrm{cm}^{3}\end{array}$} \\
\hline when analyzing the sample $V_{1}$ & 28.2526 & $3.26 \cdot 10^{-2}$ & 0.1589 & $»$ & $\propto$ & $5.18 \cdot 10^{-5}$ \\
\hline in the blank experiment $V_{0}$ & 0 & 0 & 0.1589 & » & $\propto$ & 0 \\
\hline Atomic mass of nitrogen $M, \mathrm{~g} / \mathrm{mole}$ & 14.0067 & $4.04 \cdot 10^{-6}$ & 0.3206 & $»$ & $\propto$ & $1.30 \cdot 10^{-6}$ \\
\hline $\begin{array}{l}\text { Molar concentration of } \mathrm{H}_{2} \mathrm{SO}_{4} \text {, used for the } \\
\text { titration } M, \text { mole } / \mathrm{dm}^{3}\end{array}$ & 0.1000 & $1.73 \cdot 10^{-4}$ & 44.9000 & $»$ & $\propto$ & $7.78 \cdot 10^{-3}$ \\
\hline Final titration point, $\mathrm{mV}$ & 1600 & $1.30 \cdot 10^{-4}$ & 1.0000 & $\gg$ & $\propto$ & $1.30 \cdot 10^{-4}$ \\
\hline Optimum decomposition time, $\mathrm{h}$ & 3 & $2.89 \cdot 10^{-1}$ & 0.0100 & $»$ & $\propto$ & $2.89 \cdot 10^{-3}$ \\
\hline Optimum sample mass $m_{\mathrm{s}}, \mathrm{g}$ & 1 & $5.77 \cdot 10^{-3}$ & 0.6400 & $»$ & $\propto$ & $3.70 \cdot 10^{-3}$ \\
\hline Optimum volume of distillate $V_{\mathrm{d}}, \mathrm{m}^{3}$ & 250 & 2.8930 & 0.0004 & $»$ & $\propto$ & $1.15 \cdot 10^{-3}$ \\
\hline Optimum mass of catalyst, $g$ & 2 & 0.0580 & 0.0380 & $»$ & $\propto$ & $2.19 \cdot 10^{-3}$ \\
\hline \multicolumn{7}{|l|}{ Optimum volume, $\mathrm{cm}^{3}$} \\
\hline $\mathrm{H}_{2} \mathrm{SO}_{4}$ & 10 & 0.0580 & 0.0069 & $»$ & $\propto$ & $3.98 \cdot 10^{-4}$ \\
\hline $\mathrm{H}_{2} \mathrm{O}_{2}$ & 10 & 0.0580 & 0.0430 & $»$ & $\propto$ & $2.48 \cdot 10^{-3}$ \\
\hline $\mathrm{NaOH}$ & 40 & 0.5783 & 0.0038 & $»$ & $\propto$ & $2.19 \cdot 10^{-3}$ \\
\hline \multicolumn{6}{|l|}{$u_{\text {char }}$} & 0.016 \\
\hline \multicolumn{6}{|l|}{$U(k=2, P=0.95)$} & 0.03 \\
\hline
\end{tabular}

The standard type A uncertainty [14] was calculated for $n=10$. The uncertainty of the characterization method was calculated from the formula [14]:

$$
u_{\text {char }}=\sqrt{u_{\mathrm{B}}^{2}+u_{\mathrm{A}}^{2}} .
$$

In Table 1, we show a list of the uncertainties of the results of measurements of the mass fraction of nitrogen to estimate the uncertainty of the characterization of the standard sample based on GVET 176-1-2010.

Estimation of the uncertainty due to nonuniformity. The uncertainty of the material of the standard sample of dried milk was estimated by dispersion analysis taking [12] into account, to do which the material was divided into five parts and five measurements were made with each part. As a result, we established that, in the 1-8\% range of mass fraction of nitrogen, the uncertainty characteristic $u_{h}$ is equal to $0.008 \%$.

Estimate of the uncertainty from instability. The stability of the material of the standard sample was investigated by the classical ageing method. The mass fraction of nitrogen was determined in accordance with GVET 176-1-2010 once per month, for 6 months. As a result, we established the time for which the standard sample of dried milk was still fit for purpose as being 6 months. Experiments to estimate the short-term stability of the mass fraction of nitrogen in the dried milk showed that it is possible to deliver samples under conditions of the maximum possible spread in the values of the moisture and tem- 
TABLE 2. Metrological Characteristics of GSO 9563-2010 of Whole Dried Milk (ASM-1)

\begin{tabular}{|c|c|c|}
\hline Certified characteristic & Range of values of the GSO certified characteristic, $\%$ & $\begin{array}{l}\text { Expanded uncertainty of the certified value of } \\
\text { the standard sample }(k=2), \%\end{array}$ \\
\hline Mass fraction of nitrogen ${ }^{*}$ & $2-6$ & 0.05 \\
\hline Mass fraction of moisture ${ }^{* *}$ & $2-5$ & 0.15 \\
\hline
\end{tabular}

TABLE 3. Metrological Characteristics of the CRM

\begin{tabular}{|c|c|c|c|}
\hline \multirow{2}{*}{ Metrological characteristics of the CRM } & \multicolumn{3}{|c|}{ CRM } \\
\hline & Fat-free dried milk BCR 063R & Dried whole milk BCR-380R & Fat-free dried milk BCR-380R \\
\hline Certified value - mass fraction of nitrogen & $65.3 \mathrm{mg} / \mathrm{g}$ & $28.6 \mathrm{~g} / 100 \mathrm{~g}$ & $38.2 \mathrm{~g} / 100 \mathrm{~g}$ \\
\hline Uncertainty & $0.8 \mathrm{mg} / \mathrm{g}^{*}$ & $1 \%^{* *}$ & $1 \%{ }^{* *}$ \\
\hline
\end{tabular}

perature of the surroundings for 14 days. According to [10], when calculating the uncertainty of a certified value a decision was taken to ignore the uncertainty arising from short-term instability.

Hence, the composition of dried whole milk developed in GSO 9563-2010 corresponds in its metrological characteristics (Table 2) to foreign analogs, the metrological characteristics of which are presented in Table 3. The metrological characteristics obtained enable GSO 9563-2010 to be used to monitor the accuracy of certified measurement procedures, including those standardized in [15, 16], and also for tests, checks and calibrations of express analyzers, based on different methods of measurement. In 2012-2013, GSO 9563-2010 was used for tests for the purpose of confirming the type of measuring instruments, based on the Dyum method [3], namely the nitrogen analyzers NDA 701 made by the VELP Scientifica SRL Company (Italy) and the TRUMAC made by the LECO Corporation (USA).

The significance of the development of GSO 9563-2010 is confirmed by protocol No. 41-2012 dating from May 24, 2012 of the Interstate Committee on Standardization, Metrology, and Certification of the Commonwealth of Independent States, which confirmed GSO 9563-2010 as the interstate protocol with the number MSO 1781:2012 and permitted its use in the countries of the Commonwealth without any limitations.

Conclusions. We have chosen the optimum parameters for determining the mass fraction of nitrogen based on GVET 176-1-2010. We have developed and confirmed GSO 9563-2010 on the composition of dried whole milk, certified values of which have established traceability to GVET 176-1-2010 and GET 173-2008. The metrological characteristics of GSO 9563-2010 are at the level of world standards.

\section{REFERENCES}

1. Federal Law of the Russian Federation from June 12, 2008 No. 88-FZ, On Technical Regulation on Milk and Milk Production.

2. Federal Law of the Russian Federation from June 24, 2008 No. 90-FZ, On Technical Regulation on Butter-Fat Production.

3. V. F. Volynets and M. P. Volynets, Analytical Chemistry of Nitrogen [in Russian], Nauka, Moscow (1977). 
4. M. P. Krasheninina et al., "An estimate of the accuracy of methods of processing acid-base titration curves by a potentiometric method of recording data," Zavod. Lab. Diagn. Mater., No. 12, 68-72 (2012).

5. S. V. Medvedevskikh, "Statistical models of an object and the process of measurements in the hygrometry of solid substances," Izmer. Tekhn., No. 9, 21-25 (2011); Measur. Techn., 54, No. 9, 992-998 (2011).

6. M. Yu. Medvedevskikh et al., "Additional studies of sources of uncertainty of the results of measurements of the mass fraction of moisture in grain and grain products by means of GET 173-2008 and part of the preparation for key comparisons," Izmer. Tekhn., No. 9, 66-69 (2012); Measur. Techn., 55, No. 9, 1102-1107 (2012).

7. V. V. Gorshkov et al., "State primary standard of the units of mass fraction and mass concentration of moisture in solid substances and materials," Izmer. Tekhn., No. 4, 24-27 (2010); Measur. Techn., 53, No. 4, 386-390 (2010).

8. Federal Information Fund on Ensuring Uniformity in Measurements, www.fundmetrology.ru, accessed Feb. 19, 2013.

9. MI 3174-2009, GSI. Establishment of Traceability of Certified Values of Standard Samples.

10. ISO/IEC Guide 35:2006, Natural Rubber Latex Concentrate. Determination of Mechanical Stability.

11. RMG 93-2009, GSI. Estimation of the Metrological Characteristics of Standard Samples.

12. RMG 53-2002, GSI. Standard Samples. Estimation of Metrological Characteristics Using Standards and Standard Measuring Instruments.

13. K. Derffel, Statistics in Analytical Chemistry [Russian translation], Mir, Moscow (1994).

14. EVRAKhIM/SITAK Handbook, A Quantitative Description of Uncertainty in Analytical Measurements [Russian translation], VNIIM, St. Petersburg (2002).

15. GOST 30648.2-99, Milk Products for Children's Feed. Methods of Determining Total Protein.

16. GOST 23327-98, Milk and Milk Products. A Method of Measuring the Mass Fraction of Total Nitrogen Using Kjeldahl's Method and of Determining the Mass Fraction of Protein. 